Silva dos SANTOS, FaBIAne.

Artista visual.

\title{
El Arte de tejer en Red. La fusión de lo artesanal con las nuevas tecnologías.
}

\author{
TIPO DE TRABAJO \\ Comunicación.
}

PALABRAS CLAVE

Artesanía, nuevos medios, red.

KEY WORDS

Crafts, new media, red.

RESUMEN

En el presente articulo abordamos la relación entre los lenguajes artísticos a partir del dialogo de técnicas tradicionales de la artesanía y el proceso de virtualización de la misma a partir de las últimas manifestaciones artísticas en la red y sus desdoblamientos en los nuevos medios, como en el caso de la aplicaciones móviles. Presentaremos algunas experiencias artísticas que dialogan con estas técnicas y los diferentes soportes y vehículos de presentación.

\section{ABSTRACT}

In this article we address the relationship between artistic languages dialogue from traditional craft techniques and the process of virtualization of it from the latest art forms in the network and its unfolding in new media will, as in the case of mobile applications. Present some artistic experiences that dialogue with these techniques and the different media and presentation vehicles.

\section{CONTENIDO}

La fusión entre diferentes formas de expresión es una constante en las obras artísticas en la actualidad. Últimamente muchos artistas vienen desarrollando obras donde la transición de lo artesanal a lo virtual potencializa la obra, produciendo un desdoblamiento en busca de nuevos diálogos.

La actuación artística en el ciberespacio realmente se ha expandido de forma espectacular, cualquiera que sea la plataforma que surge, de inmediato los artistas la asumen como un nuevo entorno de reflexión, discusión, promoción, etc. Esto lo vimos también con la aparición de la web 2.0, con el surgimiento de los llamados blogs, en torno a 1999, Blogger, MSN Spaces, AOL Jounals, entre otros. Según Juan Martín Prada, "a principios del siglo XXI, una nueva etapa determinada por el paso de la <<sociedad de los medios de acceso a la información>> a lo que podríamos denominar como <<sociedad de los medios personales de acceso y emisión de información $>>{ }^{1}$

Con la llegada de los blogs, muchos artistas vienen utilizando las web 2.0 como una herramienta más para desarrollar proyectos artísticos. A partir de 2015 los artistas pasan a mostrar un interés por las plataformas de blogs como medio de expresión artística. Con el adviento del blog.art, pasan a surgir los videos blogs, los blogs.collagens, etc.

\footnotetext{
${ }^{1}$ Prada, Martín Juan. Práticas Artísticas e Internet en la época de las Redes Sociales. Ediciones Akal, S.A., Madrid, 2012 .p. 159.
} 
En 2007 el colectivo Jodi realizó la obra \$BlogTitle\$, utilizando la plataforma gratuita de google blogger, en la cual procuran jugar con el texto de manera que se hace ilegible demostrando que la democratización de la Red en una farsa y que estamos condicionados a padrones. Algunas paginas del Blog fueran bloqueadas debido al sistema automatizado de blogger no reconocer las páginas ilegibles considerándolas un spamblog.

\section{FOTO}

\section{JODI}

Apps para iPhone Lo más vendido

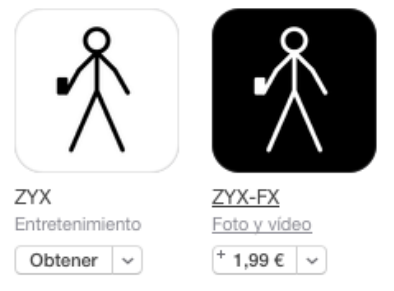

Apps para iPad

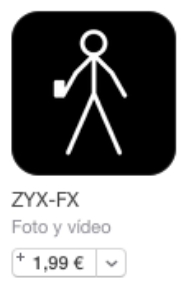

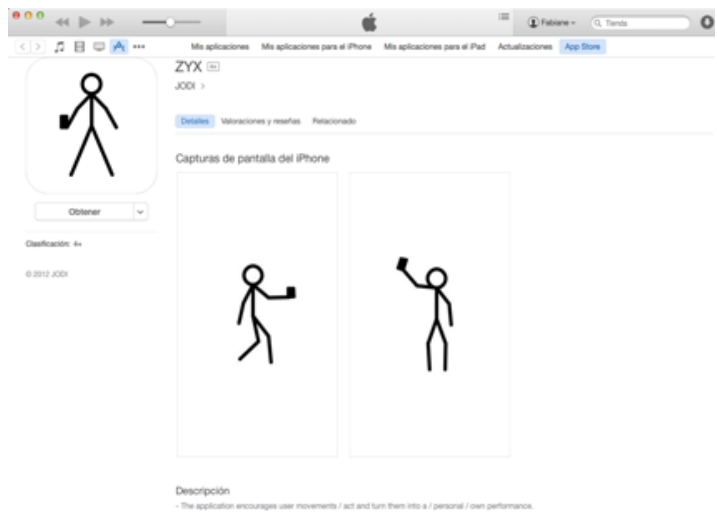

Captura de pantalla iTunes

Actualmente algunos artistas vienen desarrollando sus obras en otros formatos, en la red, que también se puede comercializar, en este caso estamos hablando de las aplicaciones desarrolladas para teléfonos móviles o sea "mobile art". Utilizado recursos de geolocalización, 3D, realidad aumentada, sistemas sonoros, etc... realizan obras donde la exclusividad ya no es una consigna, la obra se lleva encima. Las apps mobiles pasan a ser soporte, herramienta o conceptos para llevar a cabo un proyecto artístico. La pareja pionera del "New media art" Joan Heemskerk y Dirk Paesmans - JODI, han plateado un trabajo, ZYX, una aplicación para dispositivos móviles que la primer versión en 2012 era gratis, la nueva versión en 2014 tiene un coste de descarga de 1,99€. Consiste en una app que convierte el usuario en esclavo de su terminal, haciendo con que el usuario realice diversas acciones repetitivas y aparentemente inútiles. Como siempre las obras de JODI procuran hacer una crítica reflexiva sobre el uso de las tecnologías y sus efectos en la vida diaria, la obra ZYX, justo plasma ese condicionamiento de la sociedad actual con relación a las nuevas tecnologías.

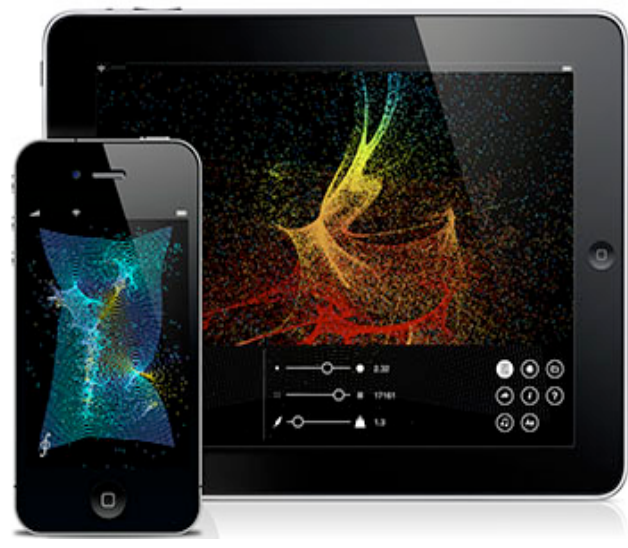

http://www.snibbestudio.com/gravilux/ 
Diversos eventos ya están siendo realizados con las aplicaciones artísticas para tablets y smartphones, en 2012 en Rio de Janeiro - Brasil, el Festival File dedicado al arte y las nuevas tecnologías, tenía organizado una sección que se llamaba Tablet dedicado exclusivamente a proyectos interactivos y artísticos realizados para dispositivos móviles, entre las obras presentadas destacó la obra "Gravilux" del artista estadounidenses Scott Snibbe, que consistía en una mezcla de juego, arte y ciencia, creada en 1998 como obra interactiva solamente disponible para galerías y museos, pero que en 2012 pasa a ser una obra disponible para aplicaciones móviles, con versiones para distintas plataformas, comercializada al valor de 1,45€. Es una obra que combina pintura, animación en tiempo real, en un ambiente que se aproxima al espacio, inspirada en la ecuación de Newton, pero no sigue las leyes propias de nuestro universo.

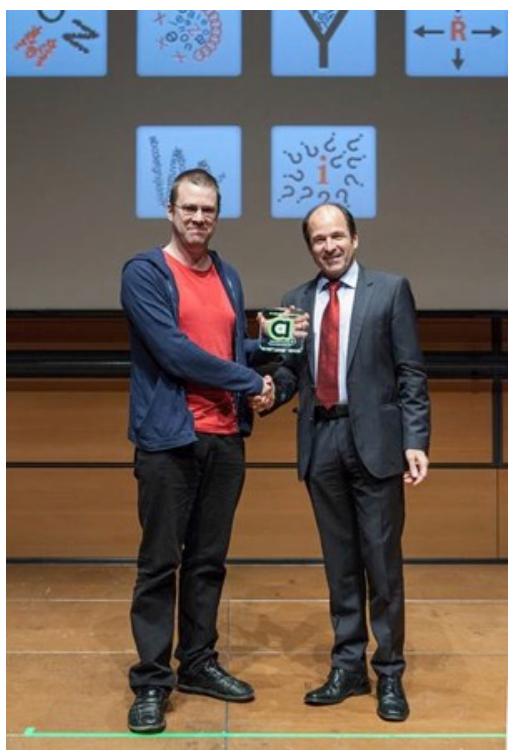

Fotografía Ganador: para su Aplicación "Konsonant" Jörg Piringer recibir con el "Premio a la Innovación Artística". (C) ZKM Karlsruhe, Foto: Felix Grünschloß. En: http://en.app-art-

award.org/retrospective/2012/

El ZKM también en ese mismo año de 2012 lanzo un concurso de App, el "AppArtAward", que premia obras realizadas en este campo. Se presentaron 84 proyectos de 13 países. La aplicación ganadora fue "Konsonat", del astriaco Jörg Piringer, disponible para plataforma Apple, consiste en una especie de maquina sonora que crea un juego con las letras y los sonidos.

Otro ejemplo de App artística es el trabajo de Clara Boj y Diego Díaz, que tiene sus trabajos relacionados a la observación de los espacios públicos y las diversas transformaciones, desde lo social, arquitectónico, funcional, etc... Uno de los proyectos que nos centramos es la App PREVISIÓN DE OLAS Y VIENTO FRIO, que forma parte del proyecto Cabanyal Archivo Vivo ${ }^{2}$ (2011), que consiste en una ficción sonora para teléfonos móviles que transcurre en el barrio del Cabanyal, en la cual el usuario se hacen partícipe de un momento cualquiera en la vida de unos vecinos de barrio. A partir del dispositivo de geolocalización, puntos calientes demarcados en un recorrido del barrio, una secuencia de audios y videos se van sucediendo mientras caminamos por el barrio. Está disponible en la página de Google play para descarga.

\footnotetext{
2 "Cabanyal Archivo Vivo es un proyecto propuesto por la asociación La Esfera Azul, coordinado por Lupe Frigols, Emilio Martinez, Bia Santos, que tiene como objetivo la puesta en valor, a través de las herramientas de la cultura, de los valores, la identidad, la memoria y el patrimonio del barrio del Cabanyal en Valencia, amenazado por los proyectos urbanísticos que pesan sobre él en la última década. Inicialmente Cabanyal Archivo Vivo, es un conjunto de acciones en el espacio real y en internet, que abarcan el ámbito pedagógico (con la realización de un material sobre el patrimonio arquitectónico y cultural del barrio), social (encuentros y mesas redondas con especialistas y asociaciones vecinales del litoral marítimo) y proyectos artísticos (una serie de propuestas a partir de conceptos de geolocalización y la edición de un número especial de la revista "la mas bella")". En: http://www.cabanyalarchivovivo.es/ acceso: 24/03/2015.
} 


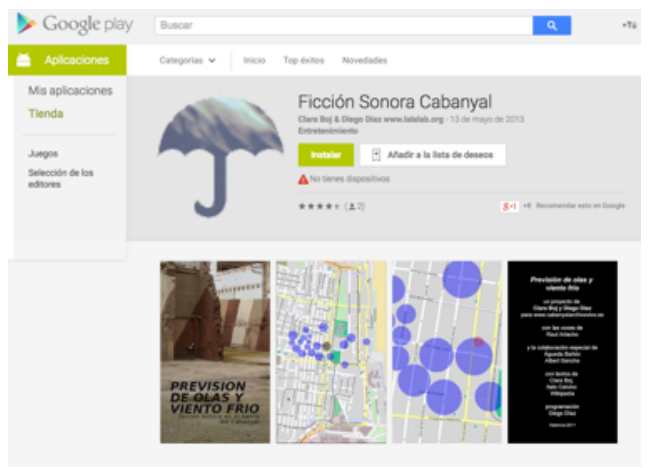

https://play.google.com/store/apps/details?id=lalalab.org.olasyViento

Otro proyecto que podemos destacar en el ámbito de derivas virtuales, a partir de narraciones, videos y sonidos en espacio real es el proyecto "Balconism" realizado en la bienal de Venecia en 2013, creación de Steve Piccolo y la curadoria de Oxana Maleeva, donde reunirán 11 artistas en una exposición virtual en los balcones de la ciudad de Venecia a partir de una aplicación disponible en App Store para iPhone y iPad.
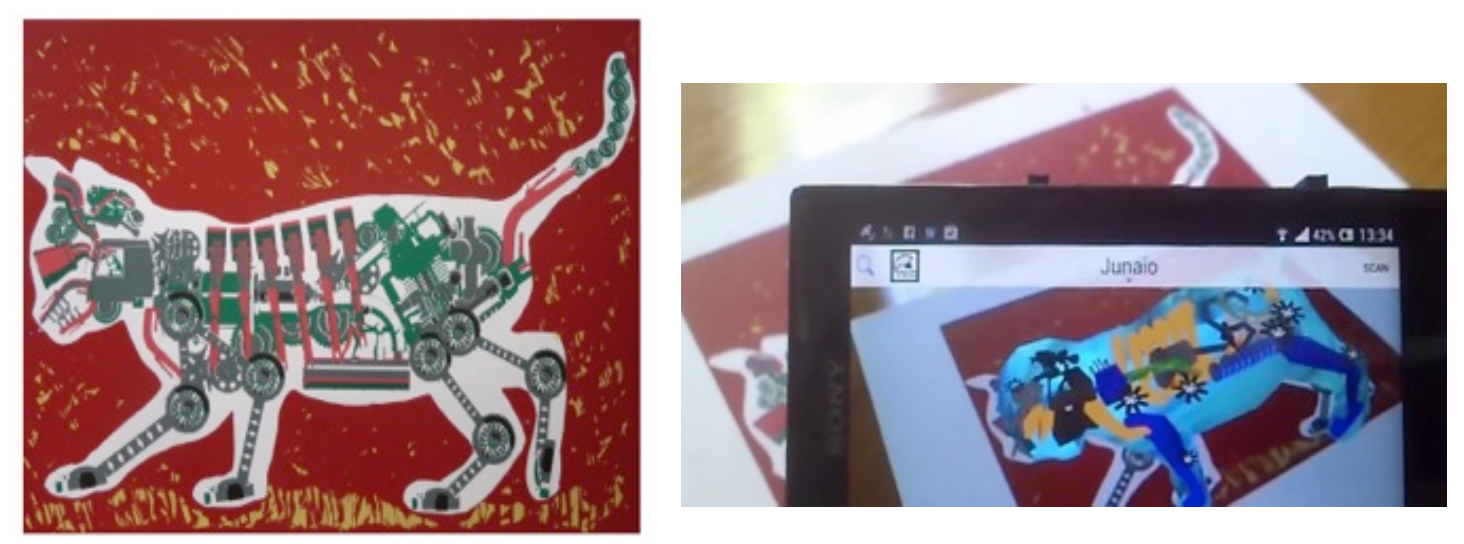

http://animalesmecanicos.webs.com/

Manusamo\&bzica es otra pareja de artista formado por Manuel Ferrer y Alena Mesarosova, que también trabajan con los nuevos medios, a través de dispositivos móviles, geolocalización, realidad aumentada, Apps, etc... Dentro de los diversos trabajos realizados por la pareja, destacamos el proyecto Animales Mecánicos ${ }^{3}$, en el cual presenta un fusión de lo artesanal con las nuevas tecnologías. A partir de grabados realizados con la técnica de la serigrafía, nos coloca a disposición una obra física que se amplia con la mezcla con las nuevas tecnologías, podemos apreciar el uso de la realidad aumentada en tecnología móvil, mezclando obra gráfica con animación 3D y sonido. Un total de 10 grabados que son comercializados y a partir de una aplicación disponible en Google play, se puede descargar gratuitamente y disfrutar de una obra interactiva. Las imágenes son animales compuestos por engranajes mecánicos, que se encargan del funcionamiento de los mismos. Al mirarlos por el dispositivo móvil, los animales cobran vida, a través de realidad aumentada, al escanear el código QR Junaio.

\footnotetext{
${ }^{3}$ Hernández, Manuel Ferrer. "Animales Mecánicos". ACTAS I Jornadas Internacionales Dibujo, Artistas y Creatividad Santa Ana de los Ríos de Cuenca, Ecuador, del 1 al 16 de Mayo de 2014. Editorial: Unidad de Cultura. Universidad de Cuenca. Ecuador. P.73.
} 


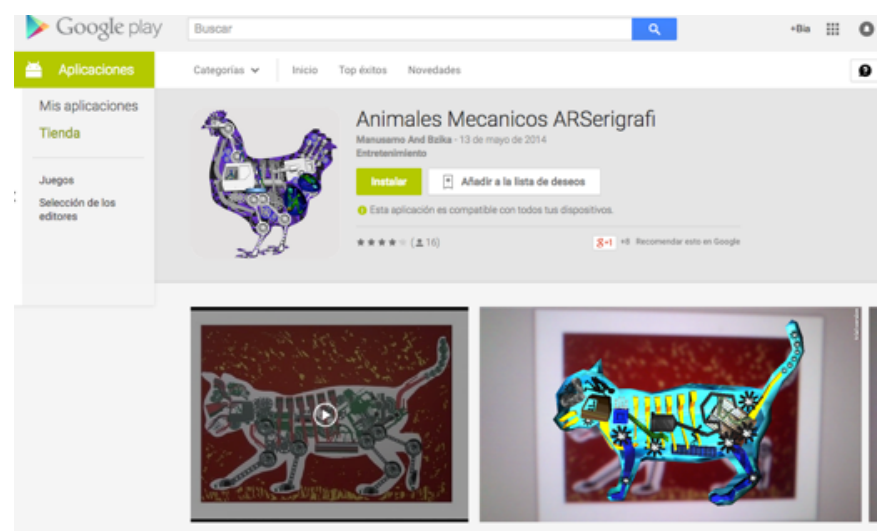

https://play.google.com/store/apps/details?id=com.manusamoandbzika.AnimalesMecanicos

"Dentro de grandes espacios históricos de tiempo se modifican, junto con toda la existencia de las colectividades humanas, el modo y manera de su percepción sensorial. Dichos modos y manera de su percepción sensorial, el medio en que acontecen, están condicionados no sólo natural, sino también históricamente" ${ }^{4}$

El arte avanza con su época y su reproducción pasa a ser una constante en nuevos formatos, cada vez más artistas utilizan las App y otros formatos como vehículos para realizar sus creaciones, no solo para promociónalas sino también para comercializarlas a muy bajo coste. Lo interesante es la posibilidad de que una obra pueda ser disfrutada por un mayor número de personas, en un proceso de reproducción desde sus propios aparatos, en cualquier parte del mundo sin necesidad de un intermediario.

\section{Experimento artístico en la red. De lo material a lo virtual}

Actualmente estamos interesados en el cruzamientos de los lenguajes artísticos en los que el proceso de creación se dan a partir del dialogo entre las técnicas tradicionales de la artesanía y el proceso de virtualización de la misma. A partir de obras matéricas que se desdoblan en un proceso hibrido a través de la Red.

Hemos desarrollado en 2011 la obra de net.art "AMBIENCE: habitaciones virtuales de navegación", tiene como referencia la propuesta artística "Ambiencia: um convite para entrar..." realizada en 1999, que consta de siete instalaciones que abordan la relación de la mujer con el hogar como un espacio privado, intimo, de reclusión y espera.

En "AMBIENCE: habitaciones virtuales de navegación", los elementos del interface se han desarrollado a partir del dibujo bordado. Los espacios virtuales son formados por interfaces realizadas a partir del dialogo de los procesos relacionados con la imagen de la mujer $y$ sus actividades privadas, como es el caso de las actividades relacionadas con las manualidades. Utilizamos el bordado como elemento que representa la relación con esa casa onírica, en la cual sus habitantes pasan a tener una nueva forma de relacionarse con el espacio público a partir de las nuevas tecnologías. En ese sentido la obra refleja ese espacio ocupado por la mujer, donde el ambiente doméstico pasa a tener nuevas aberturas y posibilidades de relacionarse con el mundo, a partir de la inclusión de las nuevas tecnologías, desde sus propias habitaciones. Está compuesta por nueve habitaciones distintas.

\footnotetext{
${ }^{4}$ Benjamin, W. "La obra de arte en la época de la reproducción técnica" en Discursos interrumpidos. Ed.Planeta Agostini, Madrid, 1994, p.
} 

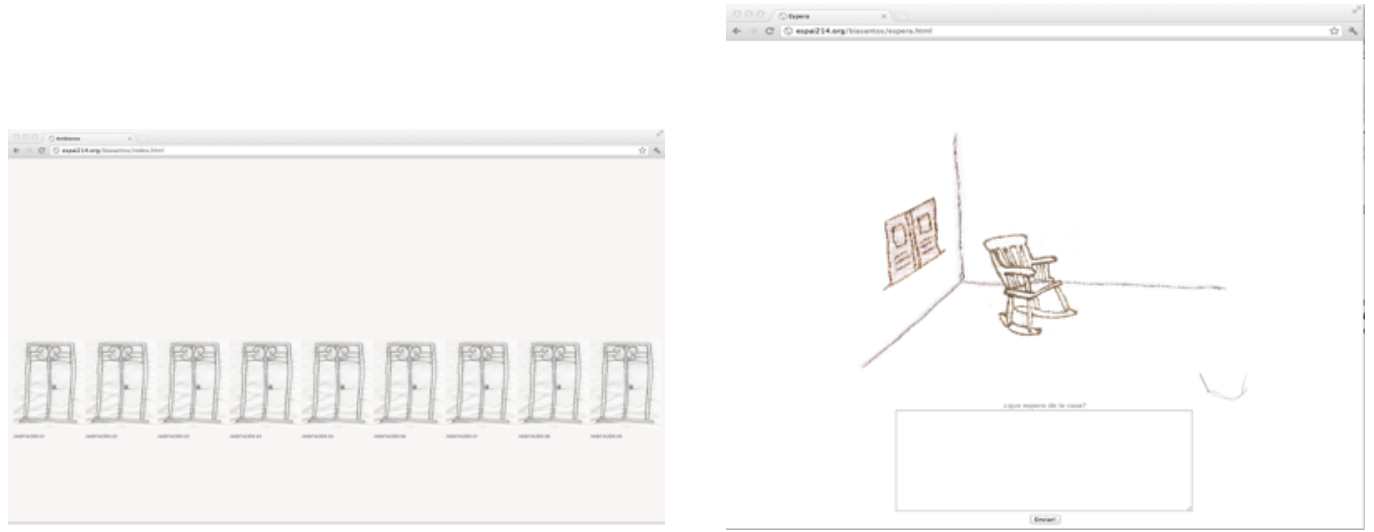

http://ambience.espai214.org/

En 2012 hemos realizado el Blog.art, la obra "Entre las cuatro paredes del hogar", en el cual procuramos utilizar la plataforma de blogger como herramienta artística para una obra en la red y romper la interface establecida, que en general son plantillas disponibles por la propia plataforma, buscamos personalizarla utilizando la tela y el bordado como elementos de navegación. Abordamos las cuestiones de género y proponiendo una reflexión sobre las distintas maneras que un mujer sufre la violencia de género. La obra está presentada a través de una plataforma de Web 2.0 (http://entrelascuatroparedesdelhogar.blogspot.com/), no como un repositorio de información sino como una obra net.art donde los hipervínculos proporcionan una navegación laberíntica hasta llegar al vínculo final que se dirigía a la pagina del extinto ministerio de la igualdad ${ }^{5}$. En general cuando hablamos de violencia machista de pronto pensamos en una agresión física. Esa es la que más repercusión tiene, es la que pone en evidencia. Pero poco a poco, entre las cuatro paredes del hogar, silenciosamente algunas mujeres sufren cada día alguno tipo de violencia sin que ellas mismas asuman esta realidad.

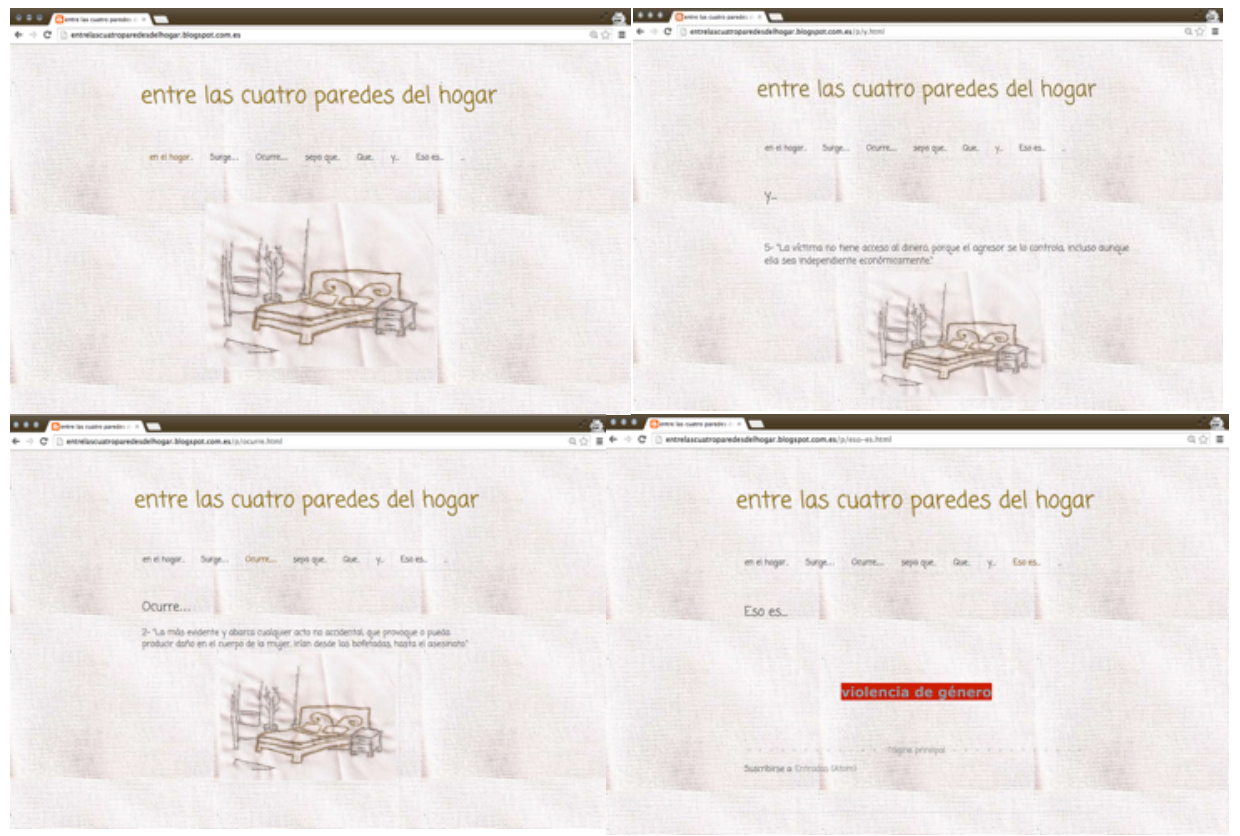

http://entrelascuatroparedesdelhogar.blogspot.com/

\footnotetext{
5 "El Ministerio de Igualdad de España se creó en 2008 durante la IX Legislatura bajo la presidencia de José Luis Rodríguez Zapatero (PSOE) para impulsar las políticas sociales recogidas en la Ley para la Igualdad y en la Ley Integral contra la Violencia sobre la Mujer, así como los programas sociales del Instituto de la Mujer y del Instituto de la Juventud. El Ministerio recogía las competencias de Igualdad que en la VIII Legislatura de España tenía el Ministerio de Trabajo y Asuntos Sociales, el cual pasó a llamarse Ministerio de Trabajo e Inmigración. El 20 de octubre de 2010, en una remodelación del Gobierno, el Ministerio fue suprimido y su estructura se integró en el Ministerio de Sanidad, Política Social e Igualdad de España1 conformando la nueva Secretaría de Estado de Igualdad". En: http://es.wikipedia.org/wiki/Ministerio de Igualdad acceso: 20/05/2014
} 
Los blogs que en general son diarios personales o un medio de comunicación en el cual la actualización del contenido es constante. Así como formato pre-establecido por la plataforma. En el blog.art "Entre las cuatro paredes del hogar" procuramos romper con esa premisa de ser un medio de información personal y interpersonal. Utilizamos la plataforma blog para realizar un proyecto de que se aproxima a una obra de net.art pero de forma gratuita (sin pagar dominio y hosting), donde el contenido tiene una implicación social relacionada con la violencia de género, sin una actualización constante.

El Web.documental ${ }^{6}$ titulado "Resistencia", realizado en 2014, es el desdoblamiento de una obra en formato dibujo bordado que consta de dos versiones, la primera el propio bordado que hace parte de la obra participativa "Cabanyal Punt a Punt" una serie de libros en tela realizado por el colectivo CraftCabanyal en la cual a partir de la imagen del bordado y la marca QR se puede acceder al web.doc; la segunda versión es el bordado digitalizado e impreso en papel a través del cual se puede acceder a web.doc a través de una marca de lectura QR. El dibujo es la fachada de una casa del barrio del Cabanyal en que se retrata su historia a través de video.blog, imágenes y geolocalización la obra es una herramienta de difusión de la problemática del barrio del Cabanyal amenazado por un proyecto urbanístico en el que los vecinos sufren la amenaza de perder sus casas. Mostramos la resistencia vecinal en defensa del patrimonio material y inmaterial del barrio.

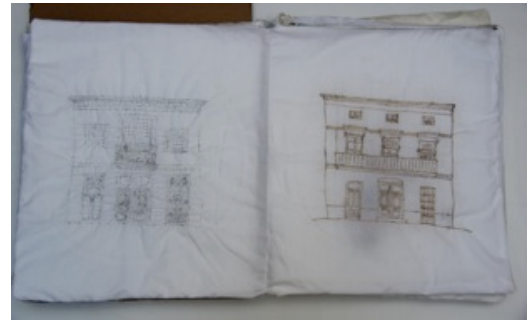

en el libros "Cabanyal punt a punt"

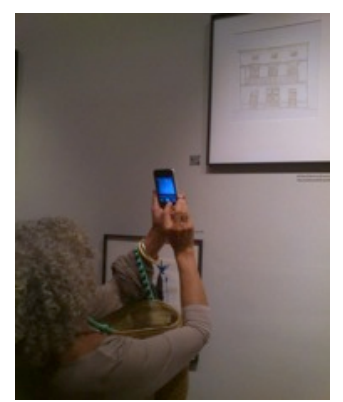

bordado digitalizado

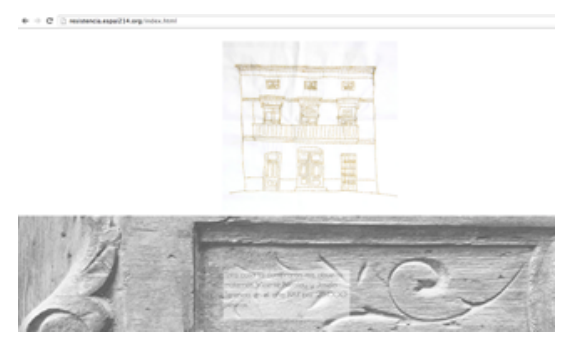

Web.Doc 


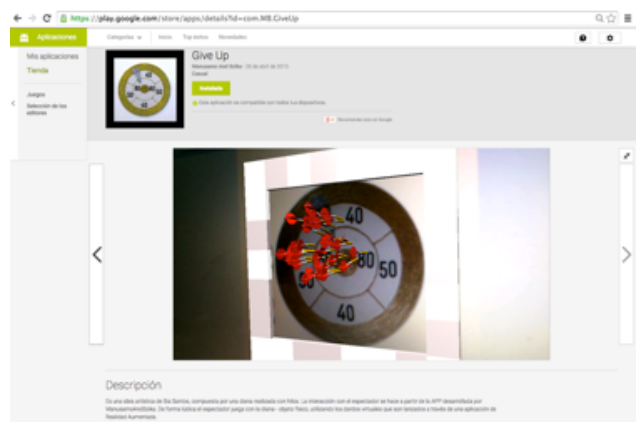

En 2015 hemos apostado por utilizar las apps como ampliación de la obra. Realizamos la obra GiveUP, donde utilizamos una diana como elemento artístico realizada con hilos dorados para bordar. Es una obra que hace una reflexión sobre el acto de renunciar, abandonar, sacrificar, parar, etc. A partir de una aplicación móvil desarrollada por Manusamo And Bzika. De forma lúdica el espectador juega con la diana - objeto físico, utilizando los dardos virtuales que son lanzados a través de la aplicación de realidad aumentada. Los dardos dorados lanzados tienen que clavarse en la propia diana, en un acto de precisión y control del aparato móvil. Es un juego que no tiene fin, juega con la provocación al usuario de parar, renunciar, abandonar, de manera simbólica representa lo que vive una parte de la sociedad actual en un sistema global competitivo.

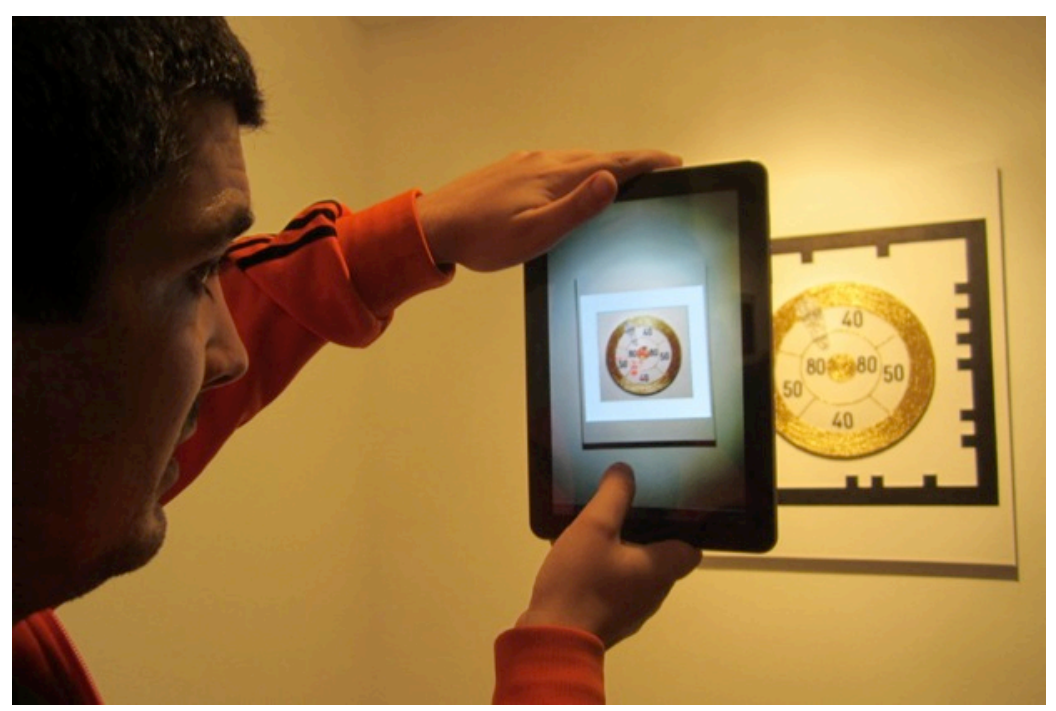

GiveUP

Foto:Bia Santos

Concluimos que en los últimos años la expresión artística viene conquistando nuevos ámbitos y nuevas formas de relacionarse con el espectador. Con el avance tecnológico la obra de arte puede llegar a cualquier hogar sin necesidad que el espectador tenga que se desplazar a una galería o a un museo. La obra se desdobla en un proceso hibrido a través de pantallas o aparatos móviles en un proceso constante de relación artista - obra - espectador.

Las obras realizadas por lo que podemos considerar baja tecnología, procuran explorar diferentes formas de expresar, de comunicar y de desdoblar un trabajo artístico a través de los nuevos medios. Se busca una autonomía por parte del propio artista que proporciona una nueva forma de relacionarse con su obra y con el espectador, al margen del llamado mercado del arte. Esa autonomía hace posible proyectos colaborativos comprometidos socialmente, donde la interacción y colaboración artista y espectador se hace una constante. 


\section{FUENTES REFERENCIALES.}

Benjamin, W. “La obra de arte en la época de la reproducción técnica” en Discursos interrumpidos. Ed.Planeta Agostini, Madrid, 1994

Brea, José Luis. La era postmedia. Acción comunicativa, prática (post)artísticas y dospositivos neomediales. Consorcio Salamanca, Salamanca, 2002.

Baigorri Ballarín, Laura. NET.ART. Prácticas estéticas y políticas en la red. Editorial: Universitat de Barcelona. Publicacion. 2006

Hernández, Manuel Ferrer. "Animales Mecánicos". ACTAS I Jornadas Internacionales Dibujo, Artistas y Creatividad Santa Ana de los Ríos de Cuenca, Ecuador, del 1 al 16 de Mayo de 2014. Editorial: Unidad de Cultura. Universidad de Cuenca. Ecuador.

Prada, Martín Juan. Práticas Artísticas e Internet en la época de las Redes Sociales. Ediciones Akal, S.A., Madrid, 2012

Prado, Gilbertto. “Estudo e Criação de Site da Arte na Internet”, Encontro Nacional da ANPAP. Anais, São Paulo, p. 296-303, 1997.

Carrillo, José. Arte en la Red. Madrid: Arte Cátedra, 2004.

Plant, Sadie. Mulher Digital: o feminino e as novas tecnologias. Rio de Janeiro: Editora Rosa dos Tempos, 1999.

Virilio, Pal. O Espaço Crítico. Rio de Janeiro: Ed. 34, 1993.

Zafra. Remedios. Un cuarto propio conectado. Fórcola Ediciones, Madrid, 2010.

\section{Electrónico}

Bosco, Roberta y Caldana, Stefano. AppArtAward: las mejores aplicaciones artísticas para tabletas ya tienen sus

Oscar.24 de septiembre de 2012. En: http://blogs.elpais.com/arte-en-la-edad-silicio/2012/09/appartaward-las-mejores-aplicacionesartisticas-para-tabletas-ya-tienen-sus-oscar.html. Acceso: 13/04/2014

. Arte de bolsillo. 25 de febrero de 2013. En: http://blogs.elpais.com/arte-en-la-edad-silicio/2013/02/arte-de-bolsillo.html. Acceso: $18 / 04 / 2014$

Valiosos laboratorios artísticos de bolsillo. 29 de agosto de 2013. En: http://blogs.elpais.com/arte-en-la-edadsilicio/2013/08/valiosos-laboratorios-artisticos-de-bolsillo.html

Cabanyal Archivo Vivo. En: http://www.cabanyalarchivovivo.es/ acceso: 24/03/2015

Documental-web. En: http://es.wikipedia.org/wiki/Documental_web. Acceso:24/03/2015

Ministerio de la igualdad. En: http://es.wikipedia.org/wiki/Ministerio_de_lgualdad acceso: 20/05/2014 